TRANSACTIONS OF THE

AMERICAN MATHEMATICAL SOCIETY

Volume 361, Number 6, June 2009, Pages 3325-3341

S 0002-9947(08)04750-8

Article electronically published on December 31, 2008

\title{
THE $K$-THEORY OF TORIC VARIETIES
}

\author{
G. CORTIÑAS, C. HAESEMEYER, MARK E. WALKER, AND C. WEIBEL
}

\begin{abstract}
Recent advances in computational techniques for $K$-theory allow us to describe the $K$-theory of toric varieties in terms of the $K$-theory of fields and simple cohomological data.
\end{abstract}

\section{INTRODUCTION}

In this paper, we revisit the $K$-theory of toric varieties, using the new perspective afforded by the recent papers [18, 2], 3. These papers provide a new technique for computations of the $K$-theory of a singular algebraic variety $X$ over a field of characteristic 0 , in terms of the homotopy $K$-theory of $X$ and cohomological data: the cyclic homology of $X$ and the $c d h$-cohomology of the sheaves $\Omega^{p}$ of Kähler differentials.

The homotopy $K$-theory $K H_{*}(X)$ of an affine toric variety is just the algebraic $K$-theory of a Laurent polynomial ring, and is well understood. Even when $X$ is a non-affine toric variety, $K H_{*}(X)$ is tractable; we show in Proposition [5.6 that it is a summand of $K_{*}(X)$. This allows us to give a short proof in Proposition 5.7 of Gubeladze's classical theorem (in [1]) that $K_{0}(X)=\mathbb{Z}$ for affine $X$.

This reduces the problem of understanding $K_{*}(X)$ to that of understanding the cyclic homology of $X$ and its $c d h$-cohomology. Because toric varieties admit resolutions of singularities that are formed in a purely combinatorial manner, it turns out this is indeed an accessible problem.

The main goal of this paper is to use these new techniques to give a streamlined approach to two of Gubeladze's recent results concerning the $K$-theory of toric varieties: examples of toric varieties with "huge" Grothendieck groups 14 and his "Dilation Theorem" (verifying the "nilpotence conjecture") 15. Our proof of this theorem is considerably shorter than the original. On the other hand, our approach and Gubeladze's are cousins in the sense that they have a common ancestor: Cortiñas' verification of the KABI conjecture [1].

Since varieties are locally smooth in the $c d h$-topology, it is not surprising that the $c d h$-fibrant version of cyclic homology is strongly related to the $c d h$-cohomology of the sheaf $\Omega^{p}$ of Kähler differentials. Theorem 4.1 below shows that, for a toric

Received by the editors September 14, 2007.

2000 Mathematics Subject Classification. Primary 19D55, 14M25, 19D25.

Key words and phrases. Algebraic $K$-theory, toric varieties.

The first author's research was partially supported by FSE and by grants ANPCyT PICT 03-12330, UBACyT-X294, JCyL VA091A05, and MEC MTM00958.

The third author's research was supported by NSF grant DMS-0601666.

The fourth author's research was supported by NSA grant MSPF-04G-184 and the Oswald Veblen Fund.

(C)2008 American Mathematical Society Reverts to public domain 28 years from publication 
variety $X$, the $c d h$-cohomology of $\Omega^{p}$ is computed by the Zariski cohomology of Danilov's sheaf of differentials $\tilde{\Omega}_{X}^{q}$. Since the global sections of $\Omega_{X}^{p}$ and $\tilde{\Omega}_{X}^{p}$ can be computed explicitly for toric varieties, it is easy to find examples of toric varieties with huge Grothendieck groups; see Example 5.10.

Gubeladze's Dilation Theorem (stated and proven in Corollary 6.10 below) asserts, roughly speaking, that after inverting the action of "dilations," the $K$-theory of a toric variety becomes homotopy invariant. Our Theorem 6.6 shows that, after inverting the action of dilations, the global sections of $\tilde{\Omega}_{X}^{q}$ agree with the Hochschild homology groups $H H_{q}(X)$. By the technique of [2], this quickly leads to our new proof of Gubeladze's theorem.

Notation. Throughout this paper, we will adhere to the following notation. Let $N$ be a free abelian group of rank $n<\infty$ and let $M=N^{*}=\operatorname{Hom}(N, \mathbb{Z})$. Define $N_{\mathbb{R}}=N \otimes_{\mathbb{Z}} \mathbb{R}$ and $M_{\mathbb{R}}=\operatorname{Hom}_{\mathbb{R}}\left(N_{\mathbb{R}}, \mathbb{R}\right) \cong M \otimes_{\mathbb{Z}} \mathbb{R}$. For $m \in M_{\mathbb{R}}, n \in N_{\mathbb{R}}$, let $\langle m, n\rangle$ denote the value of $m$ at $n$. Finally, let $k$ denote a field of characteristic 0 .

\section{REVIEW OF TORIC VARIETIES}

The material in this section may be found in standard texts, such as 9 or $[5$.

A strongly convex rational cone in $N_{\mathbb{R}}$ is a subset $\sigma \subset N_{\mathbb{R}}$ that is a cone spanned by finitely many vectors in $N$ and that contains no lines. That is, $\sigma=\mathbb{R}_{\geq 0} v_{1}+$ $\cdots+\mathbb{R}_{\geq 0} v_{k}$ for some $v_{1}, \ldots, v_{k} \in N \subset N_{\mathbb{R}}$, and whenever both $u$ and $-u$ belong to $\sigma$, we must have $u=0$. Given such a cone $\sigma$, let $\sigma^{\vee} \subset M_{\mathbb{R}}$ denote the dual cone, defined to consist of those $m \in M_{\mathbb{R}}$ such that $\langle m,-\rangle \geq 0$ on $\sigma$. Note that $\sigma^{\vee} \cap M$ is the abelian monoid (under addition of functions) of linear functions with integer coefficients on $N_{\mathbb{R}}$ whose restrictions to $\sigma$ are nowhere negative. A face of $\sigma$ is a subset $\tau$ of the form

$$
\sigma(m)=\{n \in \sigma \mid\langle m, n\rangle=0\}
$$

for some $m \in \sigma^{\vee}$. Observe that a face of a strongly convex rational cone is again a strongly convex rational cone. We write $\tau \prec \sigma$ to indicate that $\tau$ is a face of $\sigma$.

Recall that $k$ denotes a field of characteristic zero.

The affine toric $k$-variety associated to a strongly convex rational cone $\sigma$ is $U_{\sigma}=\operatorname{Spec} k\left[\sigma^{\vee} \cap M\right]$. We write elements of the monoid ring $k\left[\sigma^{\vee} \cap M\right]$ as $k$-linear combinations of the set of formal symbols $\left\{\chi^{m} \mid m \in \sigma^{\vee} \cap M\right\}$, so that multiplication in this ring is given on this $k$-basis by $\chi^{m} \cdot \chi^{m^{\prime}}=\chi^{m+m^{\prime}}$.

A fan $\Delta$ in $N_{\mathbb{R}}$ is a finite collection of strongly convex rational cones in $N_{\mathbb{R}}$ such that (1) any face of a cone in $\Delta$ is again in $\Delta$ and (2) the intersection of any two cones in $\Delta$ is a face of each. If $\tau$ is a face of $\sigma$, then $U_{\tau} \rightarrow U_{\sigma}$ is an open immersion, because the evident map $k\left[\sigma^{\vee} \cap M\right] \rightarrow k\left[\tau^{\vee} \cap M\right]$ is given by inverting a finite number of the $\chi^{m}$. It follows that for any fan $\Delta$, we may form a scheme $X(\Delta)$ by patching together the affine schemes $U_{\sigma}$ corresponding to cones $\sigma$ along the open subschemes associated to their intersections.

We call $X(\Delta)$ the toric variety associated to $\Delta$.

Orbits. We write $T_{N}=\operatorname{Spec} k[M]$ for the $n$-dimensional torus associated to $N$. Observe that $T_{N}$ acts on each $U_{\sigma}$ - equivalently, the $\operatorname{ring} k\left[\sigma^{\vee} \cap M\right]$ is naturally $M$-graded with the weight $m$ part being $k \cdot \chi^{m}$ if $m \in \sigma^{\vee}$, and 0 if $m \notin \sigma^{\vee}$. Since these actions are compatible, the torus $T_{N}$ acts on $X(\Delta)$.

The orbits of this action are tori and are in 1-1 correspondence with the cones of $\Delta$; thus $X(\Delta)$ is the disjoint union of the orbits $\operatorname{orb}(\tau)$ corresponding to the $\tau \in \Delta$. 
To describe the orbit for $\tau$, let $\mathbb{Z}(\tau \cap N)$ denote the subgroup of $N$ generated by $\tau \cap N$, and let $\bar{N}$ be the free abelian group $N / \mathbb{Z}(\tau \cap N)$. Then $\operatorname{orb}(\tau) \cong T_{\bar{N}}$. Note that the orbit corresponding to the minimal cone $\{0\}$ is the dense open orb $(0)=U_{0}$ and is naturally isomorphic to $T_{N}$.

We write $V_{\Delta}(\sigma)$ for the closure of $\operatorname{orb}(\sigma)$ in $X(\Delta)$. The orbits in $V_{\Delta}(\sigma)$ are indexed by the star of $\sigma, \operatorname{Star}_{\Delta}(\sigma)$, defined as the set of cones in $\Delta$ containing $\sigma$ :

$$
V_{\Delta}(\sigma)=\coprod_{\sigma \prec \tau} \operatorname{orb}(\tau) .
$$

Each orbit-closure $V_{\Delta}(\sigma)$ has the structure of a toric variety. To see this, let $\bar{N}=$ $N / \mathbb{Z}(\sigma \cap N)$. Then $\{\bar{\epsilon} \mid \sigma \prec \epsilon\}$ forms a fan in $\bar{N}_{\mathbb{R}}$, and the corresponding toric variety is $V_{\Delta}(\sigma)$. The torus $T_{\bar{N}}$ is a quotient of $T_{N}$ and the inclusion $V_{\Delta}(\sigma) \subset X(\Delta)$ is $T_{N}$-equivariant, the action of $T_{N}$ on $V_{\Delta}(\sigma)$ being induced by the quotient map $T_{N} \rightarrow T_{\bar{N}}$. If $\epsilon$ is a maximal cone of $\Delta$ and $\sigma$ is a face of $\epsilon$, the closed immersion $V_{\Delta}(\sigma) \cap U_{\epsilon} \hookrightarrow U_{\epsilon}$ is given by the ring surjection

$$
\operatorname{Spec} k\left[\epsilon^{\vee} \cap M\right] \rightarrow \operatorname{Spec} k\left[\epsilon^{\vee} \cap M \cap \sigma^{\perp}\right],
$$

sending $\chi^{m}$ to 0 if $m \notin \sigma^{\perp}$ and to $\chi^{m}$ if $m \in \sigma^{\perp}$.

It is useful to regard the open complement of $V_{\Delta}(\sigma)$ in $X(\Delta)$ as the toric variety corresponding to the largest sub-fan of $\Delta$ in $N_{\mathbb{R}}$ that does not contain $\sigma$.

Every toric variety is normal, but need not be smooth. A toric variety $X(\Delta)$ is smooth if and only if, for every cone $\sigma$ in the fan $\Delta$, the minimal lattice points along the 1-dimensional faces (rays) of $\sigma$ form part of a $\mathbb{Z}$-basis of $N$. In particular, in order for $X(\Delta)$ to be smooth, the set of rays of each cone must be $\mathbb{R}$-linearly independent (such a cone is said to be simplicial).

Resolution of singularities. We will need a detailed description of resolutions of singularities for toric varieties, which we now recall from [9]. If $v \in N$ is contained in one (or more) of the cones of $\Delta$, one may subdivide $\Delta$ by the ray $\rho=\mathbb{R}_{\geq 0} v$ through $v$ to form a new fan $\Delta^{\prime}$ in $N_{\mathbb{R}}$ as follows: If $\tau \in \Delta$ does not contain $\rho$, then $\tau$ is also a cone of $\Delta^{\prime}$. For each cone $\tau \in \Delta$ containing $\rho$ and for each face $\nu$ of $\tau$ not containing $\rho, \Delta^{\prime}$ contains the cone spanned by $\rho$ and $\nu$ :

$$
\tilde{\nu}:=\nu+\mathbb{R}_{\geq 0} \rho .
$$

Finally, $\rho$ itself belongs to $\Delta^{\prime}$. Thus if $\sigma \in \Delta$ is the minimal cone of $\Delta$ containing $\rho$, then $\Delta^{\prime}$ is the disjoint union of $\Delta \backslash \operatorname{Star}_{\Delta}(\sigma)$ and $\operatorname{Star}_{\Delta^{\prime}}(\rho)$.

There is a map of toric varieties $X^{\prime}=X\left(\Delta^{\prime}\right) \rightarrow X=X(\Delta)$, and it is proper, birational, and equivariant with respect to the action of the torus $T_{N}$. Starting with any toric variety $X(\Delta)$, one can arrive at a desingularization of $X(\Delta)$ by performing a finite number of subdivisions of this type.

Suppose $\Delta^{\prime}$ is the fan obtained by subdividing $\Delta$ by inserting a ray $\rho$, and let $\sigma \in \Delta$ be the minimal cone in $\Delta$ containing $\rho$. Then the description of the orbitclosures given above makes it clear that

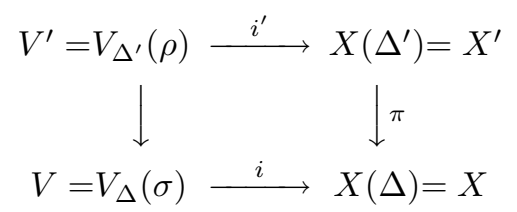

is an abstract blow-up square. That is, this a pull-back square in which the horizontal arrows are closed immersions and the map on open complements is an 
isomorphism:

$$
X\left(\Delta^{\prime}\right) \backslash V_{\Delta^{\prime}}(\rho) \stackrel{\cong}{\longrightarrow} X(\Delta) \backslash V_{\Delta}(\sigma) .
$$

As with any abstract blow-up, the maps $\left\{X\left(\Delta^{\prime}\right) \rightarrow X(\Delta), V_{\Delta}(\sigma) \rightarrow X(\Delta)\right\}$ form a covering for the $c d h$-topology. Recall that the torus $T_{N}$ acts on each variety in the above square and each map in this square is $T_{N}$-equivariant.

\section{DANILOV'S SHEAVES $\tilde{\Omega}^{p}$}

In this section, we introduce the coherent sheaves $\tilde{\Omega}_{X}^{p}$, first defined by Danilov [5. 4.2]. We will see in the next section that their Zariski cohomology groups turn out to give the $c d h$-cohomology groups of $\Omega^{p}$.

Given a fan $\Delta$, let $\Delta(1)$ denote the collection of rays in $\Delta$; the 1-skeleton of $\Delta$ is the fan $\Delta(1) \cup\{0\}$ and its toric variety $X^{(1)}$ lies in the smooth locus of $X(\Delta)$.

Definition 3.1. For a toric $k$-variety $X=X(\Delta)$ defined by a fan $\Delta$ in $N_{\mathbb{R}}$, we define $\tilde{\Omega}_{X}^{p}$ to be the coherent sheaf on $X$ fitting into the exact sequence

$$
0 \rightarrow \tilde{\Omega}_{X}^{p} \rightarrow \mathcal{O}_{X} \otimes_{\mathbb{Z}} \bigwedge^{p}(M) \stackrel{\delta}{\longrightarrow} \bigoplus_{\rho \in \Delta(1)} \mathcal{O}_{V_{\Delta}(\rho)} \otimes_{\mathbb{Z}} \bigwedge^{p-1}\left(M \cap \rho^{\perp}\right) .
$$

The component of the map $\delta$ indexed by $\rho$ sends $f \otimes\left(m_{1} \wedge \cdots \wedge m_{p}\right)$ in $\mathcal{O}_{X} \otimes \wedge_{\mathbb{Z}}^{p}(M)$ to

$$
i^{*}(f) \otimes\left(\sum_{i}(-1)^{i}\left\langle m_{i}, n_{\rho}\right\rangle m_{1} \wedge \cdots \wedge \hat{m}_{i} \wedge \cdots \wedge m_{p}\right),
$$

where $i: V_{\Delta}(\rho) \hookrightarrow X$ is the canonical closed immersion, and $n_{\rho} \in N$ is the minimal lattice point on $\rho$. By convention, $\tilde{\Omega}_{X}^{0}=\mathcal{O}_{X}$.

On the affine $U_{\sigma}$, the ring $\mathcal{O}\left(U_{\sigma}\right)$ is $M$-graded, so the sections of $\mathcal{O}_{X} \otimes \bigwedge^{p} M$ are $M$-graded with $\bigwedge^{p} M$ in weight 0 ; the weight $m$ summand is $k \cdot \chi^{m} \otimes \bigwedge^{p} M$ if $m \in \sigma^{\vee}$. Since $\delta$ is graded, it follows that each $\tilde{\Omega}_{X}^{p}\left(U_{\sigma}\right)$ is $M$-graded.

Remark 3.2. Sections of $\tilde{\Omega}_{X}^{1}$ may be considered as differential forms on $X$, with $1 \otimes m$ corresponding to the form $d \log \left(\chi^{m}\right)=d \chi^{m} / \chi^{m}$. On a non-singular cone $\sigma$, we may identify $\mathcal{O} \otimes \bigwedge^{p} M$ with the locally free sheaf $\Omega^{p}(\log D)$ of differentials with logarithmic poles along $D=\bigcup V(\rho)$. This identifies the map $\delta$ with the residue map, so we have $\left.\left.\Omega^{p}\right|_{U_{\sigma}} \cong \tilde{\Omega}^{p}\right|_{U_{\sigma}}$.

As shown by Danilov [5, 4.3], the sheaf $\tilde{\Omega}_{X}^{p}$ is naturally isomorphic to $j_{*}\left(\Omega_{U}^{p}\right)$, where $j: U \hookrightarrow X$ is the immersion of the open subscheme $U$ of smooth points of $X$. Applying Remark 3.2 to $X^{(1)} \hookrightarrow U$, we see that $\tilde{\Omega}_{X}^{p}=j_{*}^{(1)}\left(\Omega_{X^{(1)}}^{p}\right)$, where $j^{(1)}: X^{(1)} \hookrightarrow X$ is the evident open immersion.

We will need an explicit description of the $M$-grading on $\tilde{\Omega}^{1}$, or rather on the module of sections $\tilde{\Omega}^{1}\left(U_{\sigma}\right)$ over an affine toric variety $U_{\sigma}$. (See [5, 4.2.3].) When $m \in \sigma^{\vee} \cap M$, its weight $m$ summand is the subspace $\tilde{\Omega}^{1}\left(U_{\sigma}\right)_{m}=k \cdot \chi^{m} \otimes\left(M \cap \sigma(m)^{\perp}\right)$ of the weight $m$ summand $k \cdot \chi^{m} \otimes M$ of $\mathcal{O}\left(U_{\sigma}\right) \otimes M$. Here $\sigma(m)^{\perp}$ is the orthogonal complement of the face $\sigma(m)$ of $\sigma$ defined in (2.1) by the vanishing of $m$ : For $m \notin \sigma^{\vee}, \tilde{\Omega}^{1}\left(U_{\sigma}\right)_{m}=0$ because $\mathcal{O}\left(U_{\sigma}\right)_{m}=0$. More generally, we have for $m \in M$ and $p \geq 0$

$$
\tilde{\Omega}_{X}^{p}\left(U_{\sigma}\right)_{m}= \begin{cases}k \cdot \chi^{m} \otimes \bigwedge^{p}\left(M \cap \sigma(m)^{\perp}\right) & \text { if } m \in \sigma^{\vee}, \\ 0 & \text { if } m \notin \sigma^{\vee} .\end{cases}
$$


It is instructive to compare (3.3) to the analogous formula for $\Omega^{p}\left(U_{\sigma}\right)$ and $H H_{p}\left(U_{\sigma}\right)$, which are graded by the submonoid $\sigma^{\vee} \cap M$ of $M$. There is a natural map from the module $\Omega_{X}^{p}$ of Kähler differentials to $\tilde{\Omega}_{X}^{p}$. On $U_{\sigma}$ it is the $M$-graded map induced by the $M$-graded map $\Omega^{p}\left(U_{\sigma}\right) \rightarrow \mathcal{O}\left(U_{\sigma}\right) \otimes \bigwedge^{p}(M)$ defined by:

$$
\chi^{m_{0}} d \chi^{m_{1}} \wedge \cdots \wedge d \chi^{m_{p}} \mapsto(1 / p !) \chi^{m} \otimes\left(m_{1} \wedge \cdots \wedge m_{p}\right), \quad m=\sum m_{i} .
$$

Recall that the orbit-closure $V(\tau)$ for the face $\tau$ is $\operatorname{Spec}\left(k\left[\sigma^{\vee} \cap M \cap \tau^{\perp}\right]\right)$. As discussed for example in [27, 9.4.15], the Hochschild homology of a commutative $\mathbb{Q}$-algebra $R$ has a natural Hodge decomposition

$$
H H_{p}(R) \cong \bigoplus_{i \leq p} H H_{p}^{(i)}(R),
$$

and there is a natural identification $H H_{p}^{(p)}(R) \cong \Omega_{R}^{p}$.

Lemma 3.5. For each $m \in \sigma^{\vee} \cap M$, let $V=V(\sigma(m))$ denote the orbit-closure for the face $\sigma(m)$ of $\sigma$. Then the closed immersion $V \subset U_{\sigma}$ induces an isomorphism $H H_{*}\left(U_{\sigma}\right)_{m} \cong H H_{*}(V)_{m}$. In particular, for all $p$

$$
\Omega_{X}^{p}\left(U_{\sigma}\right)_{m}=\Omega^{p}(V)_{m}
$$

Proof. For convenience, let us set $A=\sigma^{\vee} \cap M$ and $B=A \cap \sigma(m)^{\perp}$, so that $U_{\sigma}=\operatorname{Spec}(k[A])$ and $V(\sigma(m))=\operatorname{Spec}(k[B])$. The immersion $V \subset U_{\sigma}$ corresponds to a surjection $k[A] \rightarrow k[B]$, which is split by the evident inclusion $\iota: k[B] \rightarrow k[A]$. Hence $H H_{*}(k[B])$ is a summand of $H H_{*}(k[A])$, and it suffices to show that $\iota$ induces a surjection on the weight $m$ summand of the complex for Hochschild homology.

Now the degree $p$ part of the Hochschild complex for $k[A]$ is $k[A]^{\otimes p+1}$, so the weight $m$ summand has a basis consisting of the $\chi^{u_{0}} \otimes \chi^{u_{1}} \cdots \otimes \chi^{u_{p}}$, where $u_{i} \in A$ and $\sum u_{i}=m$. If $n \in \sigma(m)$, then $\left\langle u_{i}, n\right\rangle \geq 0$ and $\sum_{i}\left\langle u_{i}, n\right\rangle=\langle m, n\rangle=0$. This forces each $\left\langle u_{i}, n\right\rangle=0$, i.e., $u_{i} \in B$. Hence $k[B]_{m}^{\otimes p+1}=k[A]_{m}^{\otimes p+1}$, as claimed.

Lemma 3.6. Every orbit blow-up square (2.2) determines a distinguished triangle on $X_{Z a r}$ of the form

$$
\tilde{\Omega}_{X}^{p} \rightarrow \mathbb{R} \pi_{*} \tilde{\Omega}_{X^{\prime}}^{p} \oplus i_{*} \tilde{\Omega}_{V}^{p} \rightarrow \mathbb{R} \pi_{*} i_{*}^{\prime} \tilde{\Omega}_{V^{\prime}}^{p} \rightarrow \tilde{\Omega}_{X}^{p}[1],
$$

and hence a long exact sequence of Zariski cohomology groups:

$\cdots \rightarrow H^{q}\left(X, \tilde{\Omega}^{p}\right) \rightarrow H^{q}\left(X^{\prime}, \tilde{\Omega}^{p}\right) \oplus H^{q}\left(V, \tilde{\Omega}^{p}\right) \rightarrow H^{q}\left(V^{\prime}, \tilde{\Omega}^{p}\right) \rightarrow H^{q+1}\left(X, \tilde{\Omega}^{p}\right) \rightarrow \cdots$.

Proof. We define a coherent sheaf $\tilde{\Omega}_{(X, V)}^{p}$ on $X$ by the short exact sequence

$$
0 \rightarrow \tilde{\Omega}_{(X, V)}^{p} \rightarrow \tilde{\Omega}_{X}^{p} \rightarrow i_{*} \tilde{\Omega}_{V}^{p} \rightarrow 0,
$$

and similarly a sheaf $\tilde{\Omega}_{\left(X^{\prime}, V^{\prime}\right)}^{p}$ by the sequence $0 \rightarrow \tilde{\Omega}_{\left(X^{\prime}, V^{\prime}\right)}^{p} \rightarrow \tilde{\Omega}_{X^{\prime}}^{p} \rightarrow i_{*} \tilde{\Omega}_{V^{\prime}}^{p} \rightarrow 0$ on $X^{\prime}$. Applying $\mathbb{R} \pi_{*}$ to the latter yields a morphism of distinguished triangles

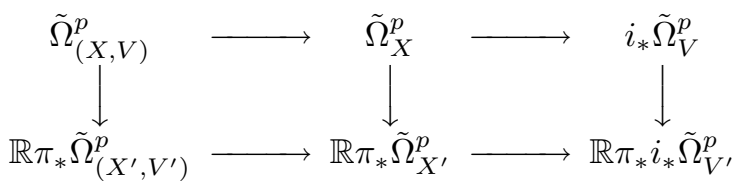

Danilov proved in [6, Prop 1.8] that the left vertical map is a quasi-isomorphism, i.e., that $\mathbb{R}^{j} \pi_{*} \tilde{\Omega}_{\left(X^{\prime}, V^{\prime}\right)}^{p}=0$ for $j>0$, and $\tilde{\Omega}_{(X, V)}^{p} \stackrel{\simeq}{\longrightarrow} \pi_{*} \tilde{\Omega}_{\left(X^{\prime}, V^{\prime}\right)}^{p}$. The distinguished triangle follows from this in a standard way. 
Remark 3.7. Danilov [5, 8.5.1] proved that if $\pi: X^{\prime} \rightarrow X$ is a morphism of toric varieties resulting from a subdivision of the fan, then $\mathcal{O}_{X} \stackrel{\simeq}{\longrightarrow} \mathbb{R} \pi_{*} \mathcal{O}_{X^{\prime}}$, i.e., $\pi_{*} \mathcal{O}_{X^{\prime}}=$ $\mathcal{O}_{X}$ and $R^{i} \pi_{*} \mathcal{O}_{X^{\prime}}=0$ for $i>0$. This proves that toric varieties have (at most) rational singularities.

\section{The $c d h$-COHOMOLOGY OF $\Omega^{p}$ FOR TORIC VARIETIES}

In this short section, we prove Theorem 4.1, that Danilov's sheaves compute the $c d h$-cohomology groups $H_{\mathrm{cdh}}^{*}\left(X, \Omega^{p}\right)$ for toric varieties.

Theorem 4.1. Let $X$ be an arbitrary toric k-variety. There is an isomorphism

$$
H_{Z a r}^{*}\left(X, \tilde{\Omega}_{X}^{p}\right) \cong H_{c d h}^{*}\left(X, \Omega^{p}\right)
$$

for all $p$, natural for morphisms of toric varieties and for the closed embedding of an orbit-closure of $X$ into $X$.

Example 4.2. The case $*=0$ of Theorem 4.1 is that $\tilde{\Omega}^{p}(X) \cong H_{\mathrm{cdh}}^{0}\left(X, \Omega^{p}\right)$. This is equivalent to Danilov's calculation [6, 1.5] that in (2.2), $\tilde{\Omega}_{X}^{p} \stackrel{\simeq}{\longrightarrow} \pi_{*} \tilde{\Omega}_{X^{\prime}}^{p}$ for all $p$.

For the proof, we recall that $H_{\mathrm{cdh}}^{*}\left(X, \Omega^{p}\right)$ is just the Zariski hypercohomology of the complex $\left.\mathbb{R} a_{*} a^{*} \Omega^{p}\right|_{X}$, where $a:(S c h / k)_{\mathrm{cdh}} \rightarrow(S c h / k)_{\text {Zar }}$ is the morphism of sites and $\left.\right|_{X}$ denotes the restriction from the big Zariski site $(S c h / k)_{\text {Zar }}$ to $X_{\mathrm{Zar}}$.

Recall that we can resolve the singularities of a toric variety via equivariant blow-up squares of the form (2.2). Iterating the orbit blow-up operations described in (2.2), as in [7, 6.2.5] we can find a smooth toric $c d h$-hypercover $\pi: Y_{\bullet} \rightarrow X$. The following Mayer-Vietoris lemma is an immediate consequence of [21, 12.1].

Lemma 4.3. For every cdh sheaf $\mathcal{F},\left.\mathbb{R} a_{*} \mathcal{F}\right|_{X} \cong \mathbb{R} \pi_{*}\left(\left.\mathbb{R} a_{*} \mathcal{F}\right|_{Y_{\bullet}}\right)$.

Proof of Theorem [4.1, As in [7, 5.2.6], Lemma 3.6 implies that the maps $\tilde{\Omega}_{X}^{p} \rightarrow$ $\mathbb{R} \pi_{*} \tilde{\Omega}_{Y_{\mathbf{\bullet}}}^{p}$ are quasi-isomorphisms. By Remark 3.2, the maps $\Omega_{Y_{\mathbf{0}}}^{p} \rightarrow \tilde{\Omega}_{Y_{\mathbf{0}}}^{p}$ are isomorphisms. Hence we have quasi-isomorphisms of complexes of Zariski sheaves on $X:$

$$
\mathbb{R} \pi_{*} \Omega_{Y_{\bullet}}^{p} \stackrel{\simeq}{\longrightarrow} \mathbb{R} \pi_{*} \tilde{\Omega}_{Y_{\bullet}}^{p} \stackrel{\simeq}{\longleftarrow} \tilde{\Omega}_{X}^{p}
$$

Now by [3, 2.5], we have $\left.\Omega_{Y_{n}}^{p} \cong \mathbb{R} a_{*} a^{*} \Omega^{p}\right|_{Y_{n}}$. Applying Lemma 4.3 to $\mathcal{F}=a^{*} \Omega^{p}$ yields

$$
\left.\mathbb{R} a_{*} a^{*} \Omega^{p}\right|_{X} \stackrel{\simeq}{\longrightarrow} \mathbb{R} \pi_{*}\left(\left.\mathbb{R} a_{*} a^{*} \Omega^{p}\right|_{Y_{\bullet}}\right) \cong \mathbb{R} \pi_{*} \Omega_{Y_{\bullet}}^{p} .
$$

Applying $H_{\text {Zar }}^{*}(X,-)$ yields $H_{\text {cdh }}^{*}\left(X, \Omega^{p}\right) \stackrel{\simeq}{\longrightarrow} H_{\text {Zar }}^{*}\left(Y_{\bullet}, \Omega^{p}\right) \cong H_{\text {Zar }}^{*}\left(X, \tilde{\Omega}^{p}\right)$, an isomorphism which is natural in the pair $Y_{\bullet} \rightarrow X$. As any two smooth toric hypercovers have a common refinement, the isomorphism $\left.\tilde{\Omega}_{X}^{p} \simeq \mathbb{R} a_{*} a^{*} \Omega^{p}\right|_{X}$ in the derived category is independent of $Y_{\bullet}$. The asserted naturality follows.

Now recall that every variety is locally smooth for the $c d h$ topology. Hence the Hochschild-Kostant-Rosenberg theorem implies that the Hochschild homology sheaf $H H_{n}$ has $a^{*} H H_{n} \cong a^{*} \Omega^{n}$. We write $\mathbb{H}_{\mathrm{cdh}}(X, H H)$ for $\mathbb{R} a_{*} a^{*}$ applied to the Hochschild complex, and $\mathbb{H}_{\mathrm{cdh}}\left(X, H H^{(t)}\right)$ for its summand in Hodge weight $t$. (See [27, 9.4.15] for a definition of the Hodge decomposition of Hochschild homology.) We write the Zariski hypercohomology of these complexes as $\mathbb{H}_{\text {cdh }}^{*}(X, H H)$ and $\mathbb{H}_{\text {cdh }}^{*}\left(X, H H^{(t)}\right)$, respectively. By [3, 2.2], $\mathbb{H}_{\mathrm{cdh}}\left(X, H H^{(t)}\right) \cong \mathbb{R} a_{*} a^{*} \Omega^{t}[t]$. Hence Theorem 4.1 translates into the following language. 
Corollary 4.4. For every toric variety $X, \mathbb{H}_{c d h}^{n}\left(X, H H^{(t)}\right) \cong H_{Z a r}^{t+n}\left(X, \tilde{\Omega}_{X}^{t}\right)$ and

$$
\mathbb{H}_{c d h}^{n}(X, H H) \cong \bigoplus_{t \geq 0} H_{Z a r}^{t+n}\left(X, \tilde{\Omega}_{X}^{t}\right)
$$

The Hochschild homology in Corollary 4.4 is taken over any field $k$ of characteristic zero. Since every toric variety $X=X_{k}$ over $k$ is obtained by base-change from a toric variety $X_{\mathbb{Q}}$ over the ground field $\mathbb{Q}$, flat base-change yields $\Omega_{X / k}^{*} \cong \Omega_{X_{\mathbb{Q}} / \mathbb{Q}}^{*} \otimes_{\mathbb{Q}} k$, and the Künneth formula yields $\Omega_{X / \mathbb{Q}}^{*}=\Omega_{X_{\mathbb{Q}} / \mathbb{Q}}^{*} \otimes_{\mathbb{Q}} \Omega_{k / \mathbb{Q}}^{*}=\Omega_{X / k}^{*} \otimes_{k} \Omega_{k / \mathbb{Q}}^{*}$. Similar formulas hold for $H H_{*}(X / \mathbb{Q})$ and hence for $\mathbb{H}_{\text {cdh }}^{*}(X, H H(-/ \mathbb{Q}))$.

We define $\tilde{\Omega}_{X / \mathbb{Q}}^{t}$ to be $j_{*} \Omega_{X / \mathbb{Q}}^{t}$. The above remarks imply that $\tilde{\Omega}_{X}^{t} \cong \tilde{\Omega}_{X_{\mathbb{Q}} / \mathbb{Q}}^{t} \otimes_{\mathbb{Q}} k$, and that there is also a Künneth formula $\tilde{\Omega}_{X / \mathbb{Q}}^{*} \cong \tilde{\Omega}_{X}^{*} \otimes_{k} \Omega_{k / \mathbb{Q}}^{*}$.

Hence we have have the following variant of the previous corollary.

Corollary 4.5. For every toric $k$-variety $X$,

$$
\mathbb{H}_{c d h}^{n}\left(X, H H^{(t)}(-/ \mathbb{Q})\right) \cong H_{Z a r}^{t+n}\left(X, \tilde{\Omega}_{X / \mathbb{Q}}^{t}\right) \cong \bigoplus_{i+j=t} H_{Z a r}^{t+n}\left(X, \tilde{\Omega}_{X}^{i}\right) \otimes_{k} \Omega_{k / \mathbb{Q}}^{j}
$$

and

$$
\mathbb{H}_{c d h}^{n}(X, H H(-/ \mathbb{Q})) \cong \bigoplus_{t \geq 0} H_{Z a r}^{t+n}\left(X, \tilde{\Omega}_{X / \mathbb{Q}}^{t}\right)
$$

\section{5. $K$-THEORY AND CYCLIC HOMOLOGY OF TORIC VARIETIES}

Recall from Section 3 that $\tilde{\Omega}_{X}^{p}$ has both a combinatorial definition and an interpretation as $j_{*} \Omega_{U}^{p}$, where $j: U \hookrightarrow X$ is the inclusion of the smooth locus. In this section, we study the exterior differentiation map $d: \tilde{\Omega}_{X}^{p} \rightarrow \tilde{\Omega}_{X}^{p+1}$ which arises as the pushforward of the de Rham differential $d: \Omega_{U}^{p} \rightarrow \Omega_{U}^{p+1}$. The following combinatorial description of this map is useful.

Lemma $5.1([5,4.4])$. The map $d: \tilde{\Omega}_{X}^{p} \rightarrow \tilde{\Omega}_{X}^{p+1}$ induced by exterior differentiation $d: \Omega_{U}^{p} \rightarrow \Omega_{U}^{p+1}$ is the $M$-graded map which in weight $m$ is $k \chi^{m} \otimes\left(m_{1} \wedge \cdots\right) \mapsto$ $k \chi^{m} \otimes\left(m \wedge m_{1} \wedge \cdots\right)$. That is, it is induced by

$$
\left(\mathcal{O}_{X}\left(U_{\sigma}\right)_{m} \otimes_{\mathbb{Z}} \bigwedge^{p} M\right) \cong \bigwedge^{p} M \stackrel{m \wedge-}{\longrightarrow} \bigwedge^{p+1} M \cong\left(\mathcal{O}_{X}\left(U_{\sigma}\right)_{m} \otimes_{\mathbb{Z}} \bigwedge^{p+1} M\right)
$$

Pushing forward the de Rham complex $\Omega_{U}^{*}$, we see that the $\tilde{\Omega}_{X}^{p}$ 's fit together to form a complex $\tilde{\Omega}_{X}^{*}$ on $X$ (the reflexive hull of the de Rham complex). There is a natural map $\Omega_{X}^{*} \rightarrow \tilde{\Omega}_{X}^{*}$ of complexes, which is an isomorphism on the smooth locus of $X$. Similarly, pushing forward the de Rham complex $\Omega_{U / \mathbb{Q}}^{*}$ from the smooth locus to $X$, we obtain a complex $\tilde{\Omega}_{X / \mathbb{Q}}^{*}$.

As in 2 and 3$], \mathbb{H}_{\mathrm{cdh}}(X, H C)$ denotes $\mathbb{R} a_{*} a^{*}$ applied to the cyclic homology cochain complex, and $\mathbb{H}_{\mathrm{cdh}}\left(X, H C^{(t)}\right)$ is its summand in Hodge weight $t$. (See [27, 9.8.14] for a definition of the Hodge decomposition of cyclic homology in characteristic 0.) The Zariski hypercohomology of these complexes is written as $\mathbb{H}_{\mathrm{cdh}}^{*}(X, H C)$ and $\mathbb{H}_{\mathrm{cdh}}^{*}\left(X, H C^{(t)}\right)$, respectively, and is called the cdh-fibrant cyclic homology of $X$.

By [3, 2.2], $\mathbb{H}_{\mathrm{cdh}}\left(X, H C^{(t)}\right) \cong \mathbb{R} a_{*} a^{*} \Omega \leq t[2 t]$, where $\Omega \leq t$ denotes the brutal ("bête") truncation of the de Rham complex. Similarly, we write $\tilde{\Omega}_{X}^{\leq t}$ for the brutal truncation of the Danilov complex $\tilde{\Omega}_{X}^{*}$. By Theorem 4.1, $\mathbb{H}_{\mathrm{cdh}}\left(X, H C^{(t)}\right) \cong \tilde{\Omega}_{X}^{\leq t}[2 t]$. 
As with Hochschild homology, the cyclic homology in the above paragraph is taken over $k$. As in the previous section, we may also consider cyclic homology taken over the ground field $\mathbb{Q}$, and we also have $\mathbb{H}_{\mathrm{cdh}}\left(X, H C^{(t)}(-/ \mathbb{Q})\right) \cong \mathbb{R} a_{*} a^{*} \Omega{ }_{/ \mathbb{Q}}^{\leq t}[2 t]$, again by [3, 2.2].

Again by Theorem 4.1, we have an isomorphism in the derived category:

$$
\mathbb{R} a_{*} a^{*} \Omega_{/ \mathbb{Q}}^{\leq t} \simeq \tilde{\Omega}_{X / \mathbb{Q}}^{\leq t}
$$

Concatenating these identifications, we have:

Proposition 5.2. If $X$ is a toric $k$-variety, the cdh-fibrant cyclic homology is given by the formulas:

$$
\mathbb{H}_{c d h}^{-n}(X, H C) \cong \bigoplus_{t \geq 0} H_{Z a r}^{2 t-n}\left(X, \tilde{\Omega}_{X}^{\leq t}\right)
$$

and

$$
\mathbb{H}_{c d h}^{-n}(X, H C(-/ \mathbb{Q})) \cong \bigoplus_{t \geq 0} H_{Z a r}^{2 t-n}\left(X, \tilde{\Omega}_{X / \mathbb{Q}}^{\leq t}\right) .
$$

Example 5.3. The case $t=0$ of Proposition 5.2 yields the formula

$$
H C_{n}^{(0)}(X)=H_{\text {Zar }}^{-n}(X, \mathcal{O}) \stackrel{\simeq}{\longrightarrow} H_{\text {cdh }}^{-n}(X, \mathcal{O})=\mathbb{H}_{\text {cdh }}^{-n}\left(X, H C^{(0)}\right) .
$$

This illustrates the interconnections between the case $p=0$ of Theorem 4.1. Danilov's calculation in Remark 3.7, and the convention that $\tilde{\Omega}_{X}^{0}=\mathcal{O}_{X}$.

These calculations tell us about the algebraic $K$-theory of toric varieties, via the following translation of [3, 1.6] into the present language.

Definition 5.4. Let $\mathcal{F}_{H C}[1]$ denote the mapping cone complex of $H C(-/ \mathbb{Q}) \rightarrow$ $\mathbb{R} a_{*} a^{*} H C(-/ \mathbb{Q})$; the indexing we use is such that there is a long exact sequence:

$$
\cdots \rightarrow H^{-n}\left(X, \mathcal{F}_{H C}\right) \rightarrow H C_{n}(X / \mathbb{Q}) \rightarrow \mathbb{H}_{\mathrm{cdh}}^{-n}(X, H C(-/ \mathbb{Q})) \rightarrow \cdots .
$$

Theorem $5.5([3,1.6])$. For every $X$ in $S c h / k$, there is a long exact sequence

$$
\cdots \rightarrow K H_{n+1}(X) \rightarrow H_{Z a r}^{-n}\left(X, \mathcal{F}_{H C}[1]\right) \rightarrow K_{n}(X) \rightarrow K H_{n}(X) \rightarrow \cdots .
$$

For toric varieties, the sequence (5.5) splits:

Proposition 5.6. For every toric variety $X, K_{*}(X) \rightarrow K H_{*}(X)$ is a split surjection. Hence

$$
K_{n}(X) \cong K H_{n}(X) \oplus H_{Z a r}^{-n}\left(X, \mathcal{F}_{H C}[1]\right) .
$$

Proof. For each affine cone $\sigma, M(\sigma):=M \cap \sigma^{\perp}$ is a free abelian monoid, so $T_{\sigma}=$ $\operatorname{Spec}(k[M(\sigma)])$ is a torus. We first claim that the inclusion

$$
i_{\sigma}: k[M(\sigma)] \longmapsto k\left[M \cap \sigma^{\vee}\right]
$$

or surjection $U_{\sigma} \rightarrow T_{\sigma}$ induces an isomorphism on $K H$-theory, i.e.,

$$
K\left(T_{\sigma}\right) \stackrel{\simeq}{\longrightarrow} K H\left(T_{\sigma}\right) \stackrel{\simeq}{\longrightarrow} K H\left(U_{\sigma}\right) .
$$

Since (5.6a) also factors as $K\left(T_{\sigma}\right) \rightarrow K\left(U_{\sigma}\right) \rightarrow K H\left(U_{\sigma}\right)$, this proves the proposition for $U_{\sigma}$.

Because $T_{\sigma}$ is regular, the first map is an isomorphism. For a suitable rational $n \in \sigma$, evaluation at $n$ is a monoid map from $M \cap \sigma^{\vee}$ to $\mathbb{N}$ with kernel $M(\sigma)$.

This gives $k\left[M \cap \sigma^{\vee}\right]$ the structure of an $\mathbb{N}$-graded algebra with $k[M(\sigma)]$ in degree zero. By [26, 1.2], $i_{\sigma}$ induces an isomorphism $K H(k[M(\sigma)]) \cong K H\left(k\left[M \cap \sigma^{\vee}\right]\right)$, as claimed. 
If $\tau$ is a face of $\sigma$, we have a commutative diagram

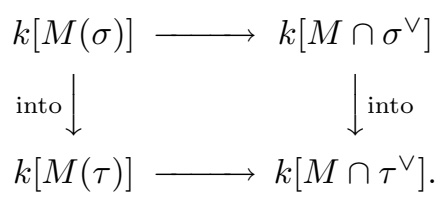

Thus the isomorphism in (5.6a) is natural in $\sigma$, for $\sigma$ a face of a fan $\Delta$, and so is the splitting of $K\left(U_{\sigma}\right) \rightarrow K H\left(U_{\sigma}\right)$. Since $K(X)$ is the homotopy limit over $\Delta$ of the $K\left(U_{\sigma}\right)$, and similarly for $K H(X)$, the homotopy limit of the splittings provides a splitting of the map $K(X) \rightarrow K H(X)$.

The sequence (5.5) is compatible with the decomposition arising from the Adams operations because the Chern character is, by [4. Thus $K_{*}^{(i)}(X)$ and $K H_{*}^{(i)}(X)$ fit into a long exact sequence with $\mathcal{F}_{H C}^{(i-1)}$. For example, it is immediate from Example 5.3 that $\mathcal{F}_{H C}^{(0)}(X)$ is acyclic, proving that $K_{*}^{(1)}(X) \cong K H_{*}^{(1)}(X)$ for toric varieties. The case $*=0$, which is a well known assertion about the Picard group of normal varieties, has the following extension:

Proposition 5.7. If $X=U_{\sigma}$ is an affine toric $k$-variety, then $K_{0}(X)=\mathbb{Z}$.

Proof. Note that the coordinate ring of $U_{\sigma}$ is graded, so $K H_{0}(X)=\mathbb{Z}$.

By Theorem [5.5, we need to show that $\mathbb{H}^{0}\left(X, \mathcal{F}_{H C}\right)=0$. Since $H C_{-1}(X)=0$, we are reduced to proving that the map

$$
H C_{0}(X) \rightarrow \mathbb{H}_{\mathrm{cdh}}^{0}(X, H C)
$$

is onto. By Proposition 5.2, the target of this map is $\bigoplus_{t \geq 0} H_{\mathrm{Zar}}^{2 t}\left(X, \tilde{\Omega}_{/ \mathbb{Q}}^{\leq t}\right)$. Since $X$ is affine, we have $H_{\mathrm{Zar}}^{2 t}\left(X, \tilde{\Omega}_{/ \mathbb{Q}}^{\leq t}\right)=0$ for all $t>0$. Finally, when $t=0$ we have

$$
H_{\mathrm{Zar}}^{0}(X, \tilde{\Omega} \underset{/ \mathbb{Q}}{\leq 0})=H_{\mathrm{Zar}}^{0}\left(X, \mathcal{O}_{X}\right)=H C_{0}(X)
$$

Remark 5.7.1. A much better version of this Proposition was proven years ago by Gubeladze [11: For a PID $R$, every finitely projective module over $R[A]$, where $A$ is a semi-normal, abelian, cancellative monoid without non-trivial units, is free. This was extended to the case where $R$ is regular by Swan 22 .

Of course, the dictionary coming from 3 via Theorem 5.5 also allows us to say something about the higher $K$-theory of toric varieties. Let $K_{n}^{(i)}(X)$ denote the weight $i$ part of $K_{n}(X) \otimes \mathbb{Q}$ with respect to the Adams operations, i.e., the eigenspace where $\psi^{k}=k^{i}$ for all $k$. We adopt the parallel notation $K H_{n}^{(i)}(X)$ for the weight $i$ part of $K H_{n}(X)$.

The absolute cotangent sheaf $\mathbb{L}_{X}$ of $X / \mathbb{Q}$ has $\mathbb{L}_{\bar{X}}^{\geq 0}=\Omega_{X / \mathbb{Q}}^{1}$ and $H^{1-n}\left(X, \mathbb{L}_{X}\right)=$ $H H_{n}^{(1)}(X / \mathbb{Q})$; see [27, 8.8.9]. There is a natural map $\mathbb{L}_{X} \rightarrow \Omega_{X / \mathbb{Q}}^{1} \rightarrow \tilde{\Omega}_{X / \mathbb{Q}}^{1}$.

Corollary 5.8. For any toric $k$-variety $X$, we have a distinguished triangle

$$
\mathcal{F}_{H C}^{(1)} \rightarrow \mathbb{L}_{X} \rightarrow \tilde{\Omega}_{X / \mathbb{Q}}^{1} \rightarrow \mathcal{F}_{H C}^{(1)}[1],
$$

and hence an isomorphism $K_{q}^{(2)}(X) \cong K H_{q}^{(2)}(X) \oplus H_{Z a r}^{2-q}\left(X, \mathbb{L}_{X} \rightarrow \tilde{\Omega}_{X / \mathbb{Q}}^{1}\right)$. 
Proof. The Zariski sheaf $H C^{(1)}$ is the mapping cone of $\mathcal{O} \rightarrow \mathbb{L}_{X}$; see [27, 9.8.18]. Since $\mathbb{R} a_{*}\left(a^{*} \mathcal{O}\right) \mid X=\mathcal{O}_{X}$ by Remark 3.7, and $\mathbb{H}_{\mathrm{cdh}}\left(X, H C^{(1)}\right) \simeq\left(\mathcal{O} \rightarrow \tilde{\Omega}_{X}^{1}\right)[2]$ by Proposition 5.2, it follows that the mapping cone $\mathcal{F}_{H C}^{(1)}$ of $H C^{(1)} \rightarrow \mathbb{H}_{\mathrm{cdh}}\left(X, H C^{(1)}\right)$ is also the mapping cone of $\mathbb{L}_{X} \rightarrow \tilde{\Omega}_{X}^{1}$. This proves the first assertion; the second assertion follows from this, Proposition [5.6 and [3, 1.6].

The techniques of [3] allow us to find examples of toric varieties with "huge" $K_{0}$ and $K_{1}$ groups, in the spirit of [25, 12] and [14. Our toric varieties will have quotient singularities because all the cones will be simplices; see [9].

Example 5.9. Let $N=\mathbb{Z}^{3}$, and let us agree to write elements of $N$ as column vectors and elements of $M \cong \mathbb{Z}^{3}$ as row vectors. Define $\tau$ to be the cone in the $x y$-plane of $N_{\mathbb{R}}=\mathbb{R}^{3}$ spanned by the vectors $e_{1}=\left[\begin{array}{l}1 \\ 0 \\ 0\end{array}\right]$ and $e_{1}+2 e_{2}=\left[\begin{array}{l}1 \\ 2 \\ 0\end{array}\right]$. Then $U_{\tau}$ is a singular, affine toric $k$-variety.

In fact, $U_{\tau}=\operatorname{Spec}\left(k[X, Y, Z] /\left(Y Z-X^{2}\right)\left[T, T^{-1}\right]\right)$, where $X=\chi^{(1,0,0)}, Y=$ $\chi^{(0,1,0)}, Z=\chi^{(2,-1,0)}$ and $T^{ \pm 1}=\chi^{(0,0, \pm 1)}$. This is because $\tau^{\vee} \cap M$ is generated by the vectors $(1,0,0),(0,1,0),(2,-1,0)$ and $(0,0, \pm 1)$.

Let $m \in M$ be the vector $(1,0,0)$. Its face is $\tau(m)=\{0\}$, so $\tau(m)^{\perp}=M$. We see from (3.3) that $\tilde{\Omega}^{1}\left(U_{\tau}\right)_{m}=k \cdot X \otimes M \cong k^{3}$. The forms $d X, X d Y / Y$ and $X d T / T$ form a basis. On the other hand, $\Omega^{1}\left(U_{\tau}\right)_{m}$ is the $k$-vector space spanned by $\chi^{u} d\left(\chi^{v}\right)$ with $u, v \in \tau^{\vee} \cap M$ satisfying $u+v=m$. It is easy to see that the only $u, v \in \tau^{\vee} \cap M$ satisfying $u+v=(1,0,0)$ are when $u, v$ is $\{(0,0,-j),(1,0, j)\}$. Thus $\Omega^{1}\left(U_{\tau}\right)_{m}$ is the 2-dimensional vector space spanned by $d X$ and $X d T / T$. It follows that $\Omega^{1}\left(U_{\tau}\right) \rightarrow \tilde{\Omega}^{1}\left(U_{\tau}\right)$ is not onto in weight $m$.

Similar reasoning shows that for $m=(1,0, c)$ we also have $\tilde{\Omega}^{1}\left(U_{\tau}\right)_{m} \cong k^{3}$ on $T^{c} d X, T^{c} X d Y / Y$ and $T^{c-1} X d T$, and that $\tilde{\Omega}^{1}\left(U_{\tau}\right)_{m}=\Omega^{1}\left(U_{\tau}\right)$ for all other $m$. (It is useful to use the fact that $\Omega^{1}\left(U_{\tau}\right)$ is a submodule of $\tilde{\Omega}^{1}\left(U_{\tau}\right)$ by [23].) Thus $\tilde{\Omega}^{1}\left(U_{\tau}\right) / \Omega^{1}\left(U_{\tau}\right) \cong k\left[T, T^{-1}\right]$. By the Künneth formula,

$$
\operatorname{coker}\left\{\Omega^{1}\left(U_{\tau} / \mathbb{Q}\right) \rightarrow \tilde{\Omega}^{1}\left(U_{\tau} / \mathbb{Q}\right)\right\} \cong \tilde{\Omega}^{1}\left(U_{\tau}\right) / \Omega^{1}\left(U_{\tau}\right) .
$$

As in Proposition [5.6, it is easy to see that $K H_{*}\left(U_{\tau}\right) \cong K_{*}\left(k\left[T, T^{-1}\right]\right)$. Hence Corollary 5.8 implies that $K_{1}^{(2)}\left(U_{\tau}\right)$ is isomorphic to a non-zero $k$-vector space:

$$
K_{1}^{(2)}\left(U_{\tau}\right) \cong H_{\mathrm{Zar}}^{1}\left(U_{\tau}, \Omega^{1} \rightarrow \tilde{\Omega}^{1}\right) \cong \tilde{\Omega}^{1}\left(U_{\tau}\right) / \Omega^{1}\left(U_{\tau}\right) \cong k\left[T, T^{-1}\right] .
$$

Example 5.10. We now extend the $\tau$ of Example 5.9 to form a fan $\Delta$ consisting of two 3-dimensional cones $\sigma_{1}, \sigma_{2}$ (together with all of their faces) such that $\sigma_{1} \cap \sigma_{2}=$ $\tau$. Specifically, let $\sigma_{1}$ and $\sigma_{2}$ be spanned by the two edges of $\tau$ together with

$$
v_{1}=\left[\begin{array}{r}
-1 \\
0 \\
+1
\end{array}\right] \quad \text { and } \quad v_{2}=\left[\begin{array}{r}
-1 \\
0 \\
-1
\end{array}\right]
$$

respectively. Let $X=X(\Delta)$, so $X=U_{\sigma_{1}} \cup U_{\sigma_{2}}$ and $U_{\tau}=U_{\sigma_{1}} \cap U_{\sigma_{2}}$. It follows from Proposition 5.6 that $K H_{0}(X)=\mathbb{Z} \oplus \mathbb{Z}$ and that

$$
K_{0}(X) \cong \mathbb{Z}^{2} \oplus H_{\text {Zar }}^{1}\left(X, \mathcal{F}_{H C}\right) .
$$

We will show that the right-hand term is non-zero; since it is a $k$-vector space, it will follow that $K_{0}(X)$ contains the additive group underlying a non-zero $k$-vector space. Taking $k$ to be uncountable, for example $k=\mathbb{C}$, we see $K_{0}(X)$ is uncountable. 
Because the singular locus of $X$ is 1-dimensional, $H^{n}\left(X, \mathbb{L}_{X}\right)=H^{n}\left(X, \Omega_{X}^{1}\right)$ for $n>0$. By Corollary 5.8

$$
K_{0}^{(2)}(X)=H_{\text {Zar }}^{1}\left(X, \mathcal{F}_{H C}\right)=H_{\text {Zar }}^{2}\left(X, \Omega^{1} \rightarrow \tilde{\Omega}^{1}\right) .
$$

From the Mayer-Vietoris sequence for the given cover of $X$, and Proposition 5.7 we see that there is an exact sequence

$$
\tilde{\Omega}^{1}\left(U_{\sigma_{1}}\right) / \Omega^{1}\left(U_{\sigma_{1}}\right) \oplus \tilde{\Omega}^{1}\left(U_{\sigma_{2}}\right) / \Omega^{1}\left(U_{\sigma_{2}}\right) \rightarrow \tilde{\Omega}^{1}\left(U_{\tau}\right) / \Omega^{1}\left(U_{\tau}\right) \rightarrow K_{0}^{(2)}(X) \rightarrow 0 .
$$

By Example 5.9, $\tilde{\Omega}^{1}\left(U_{\tau}\right) / \Omega^{1}\left(U_{\tau}\right)$ is zero except in weights $m=(1,0, c), c \in \mathbb{Z}$, where it is spanned by the forms $T^{c} X d Y / Y$. For such $m, \tau(m)=\{0\}$. If $c>0$, then $m \in \sigma_{1}^{\vee}$ and the element $\chi^{m} d Y / Y \in \tilde{\Omega}^{1}\left(U_{\sigma_{1}}\right)$ maps to $T^{c} X d Y / Y \in \tilde{\Omega}\left(U_{\tau}\right)$. If $c<0$, then $m \in \sigma_{2}^{\vee}$ and the element $\chi^{m} d Y / Y \in \tilde{\Omega}^{1}\left(U_{\sigma_{1}}\right)$ maps to $T^{c} X d Y / Y \in$ $\tilde{\Omega}\left(U_{\tau}\right)$.

We are left with the form $X d Y / Y$ in weight $m=(1,0,0)$. Since $m \notin \sigma_{i}^{\vee}$ for $i=1,2$, we have $\tilde{\Omega}^{1}\left(U_{\sigma_{1}}\right)_{m}=\tilde{\Omega}^{1}\left(U_{\sigma_{2}}\right)_{m}=0$. This proves that

$$
K_{0}^{(2)}(X) \cong \tilde{\Omega}^{1}\left(U_{\tau}\right) / \Omega^{1}\left(U_{\tau}\right)_{(1,0,0)} \cong k .
$$

As in Gubeladze's example of toric varieties with "huge" Grothendieck groups in [14, we can further extend $\Delta$ to obtain a complete fan consisting of simplicial cones $\bar{\Delta}$, so that $\bar{X}=X(\bar{\Delta})$ is a projective closure of $X$ and such that $Y=X(\bar{\Delta}-\Delta)$ is smooth. Since $Y$ and $X$ form an open cover of $\bar{X}$, we see that $K_{0}(\bar{X})$ also contains the additive group underlying a non-zero $k$-vector space.

\section{Gubeladze's Dilation Theorem}

The main goal of this section is to give a new proof of Gubeladze's Dilation Theorem 15 for the $K$-theory of monoid rings, which we obtain in Corollary 6.10 as a corollary of a version of this result valid for all toric varieties (Theorem 6.9).

For a toric variety $X=X(\Delta)$ with $\Delta$ a fan in $N_{\mathbb{R}}$ and integer $c \in \mathbb{N}$, define $\theta_{c}: X(\Delta) \rightarrow X(\Delta)$ to be the endomorphism of toric varieties induced by the endomorphism of the lattice $N$ given by multiplication by $c$. If $\sigma \subset N_{\mathbb{R}}$ is a cone, the map $\theta_{c}: U_{\sigma} \rightarrow U_{\sigma}$ of affine toric $k$-varieties is induced by the ring endomorphism of $k\left[\sigma^{\vee} \cap M\right]$ that sends $\chi^{m}$ to $\chi^{c m}$. That is, this is the map that raises all monomials to the $c$-th power. Observe that if $k=\mathbb{F}_{p}$ and $c=p$, this is precisely the Frobenius endomorphism, and it useful to think of $\theta_{c}$ as a generalization of Frobenius that exists in the category of toric varieties.

Fix a sequence $\mathfrak{c}=\left(c_{1}, c_{2}, \ldots\right)$ of integers with $c_{i} \geq 2$ for all $i$. If $F$ is a contravariant functor from toric varieties to abelian groups, we define $F^{\mathfrak{c}}$ by

$$
F(X)^{\mathfrak{c}}=\underline{\lim }\left(F(X) \stackrel{\theta_{c_{1}}^{*}}{\longrightarrow} F(X) \stackrel{\theta_{c_{2}}^{*}}{\longrightarrow} \cdots\right) .
$$

Gubeladze's Dilation Theorem asserts that the natural map $K_{*}(X) \rightarrow K H_{*}(X)$ induces an isomorphism $K_{*}(X)^{\mathfrak{c}} \rightarrow K H_{*}(X)^{\mathfrak{c}}$ for any toric variety $X$. Our proof of this theorem involves computing $H H_{q}(X)^{\mathfrak{c}}$, where $H H_{*}$ denotes Hochschild homology.

Fix a cone $\sigma$. As in the proof of Lemma 3.5, the chain complex defining the Hochschild homology of $k\left[\sigma^{\vee} \cap M\right]$ is $\sigma^{\vee} \cap M$-graded with the weight of $\chi^{m_{0}} \otimes \cdots \otimes$ $\chi^{m_{p}}$ defined to be $m_{0}+\cdots+m_{p}$, and the Hochschild homology groups of $U_{\sigma}$ are $\sigma^{\vee} \cap M$-graded $k\left[\sigma^{\vee} \cap M\right]$-modules. A fortiori, they are $M$-graded, with zero in 
weight $m$ if $m \notin \sigma^{\vee}$. Since $\theta_{c}\left(\chi^{m_{0}} \otimes \cdots\right)=\chi^{c m_{0}} \otimes \cdots, \theta_{c}$ sends the weight $m$ summand to the weight $\mathrm{cm}$ summand.

The Hochschild homology of a non-affine variety is defined by taking Zariski hypercohomology of the sheafification of the complex defined just as in the definition of $H H_{*}(R)$, but with $\mathcal{O}_{X} \otimes_{k} \cdots \otimes_{k} \mathcal{O}_{X}$ in place of $R \otimes_{k} \cdots \otimes_{k} R$ (see [24, 4.1]).

For a toric variety $X=X(\Delta)$, we may compute $H H_{*}(X)$ as follows: Let $\sigma_{1}, \ldots, \sigma_{m}$ denote the maximal cones in the fan $\Delta$. For each $1 \leq i_{0} \leq \cdots \leq i_{p} \leq m$, we may form the complex defining the Hochschild homology of the affine toric variety $U_{\sigma_{i_{0}} \cap \cdots \cap \sigma_{i_{p}}}$. We then assemble these into a bicomplex in the usual Cech manner and take the homology of the associated total complex.

Lemma 6.1. For any toric variety $X=X(\Delta)$, the groups $H H_{*}(X)$ have a natural $M$-grading, and the endomorphism $\theta_{c}$ maps the weight $m$ summand to the weight cm summand.

Proof. We have seen that the Hochschild complexes forming the columns of the bicomplex are $M$-graded. Since the ring maps are all $M$-graded, the Čech differentials are also $M$-graded. Since $H H_{*}(X)$ is the homology of an $M$-graded bicomplex, it is $M$-graded. Since the map $\theta_{c}$ sends the weight $m$ subcomplex to the weight $\mathrm{cm}$ subcomplex, it has the same effect on homology.

Remark 6.1.1. This construction implies that the Čech spectral sequence is $M$ graded:

$$
E_{p q}^{1}=\bigoplus_{i_{0}<\cdots<i_{p}} H H_{q}\left(U_{\sigma_{i_{0}} \cap \cdots \cap \sigma_{i_{p}}}\right) \Rightarrow H H_{q-p}(X) .
$$

Lemma 6.2. Set $A=\sigma^{\vee} \cap M$. If $m \in A$ lies on no proper face of $\sigma^{\vee}$, then $A+\langle-m\rangle=M$, and $k[A]\left[\chi^{-m}\right]=k[M]$.

Proof. Since $k[A]\left[\chi^{-m}\right]=k[A+\langle-m\rangle]$, it suffices to prove the first assertion, i.e., that every $t \in M$ is of the form $a-i m$ for some positive integer $i$. Fix a non-zero $n \in N$. The assumption that $m$ lies on no proper face of $\sigma^{\vee}$ implies that $\langle m, n\rangle>0$. Hence $\langle t+i m, n\rangle>0$ for $i \gg 0$. Since $\sigma \cap N$ is finitely generated, it follows that $t+i m \in A$ for $i \gg 0$, as claimed.

Lemma 6.3. The map $\theta_{c}: \Omega^{q}\left(U_{\sigma}\right)_{m} \rightarrow \Omega^{q}\left(U_{\sigma}\right)_{c m}$ is multiplication by $c^{q} \chi^{(c-1) m}$.

Proof. When $\sum u_{i}=m, \theta_{c}$ takes $\omega=\chi^{u_{0}} d \chi^{u_{1}} \wedge \cdots \wedge d \chi^{u_{q}}$ to $c^{q} \chi^{(c-1) m} \omega$.

Remark 6.3.1. The same proof shows that the map $\theta_{c}: \tilde{\Omega}^{q}\left(U_{\sigma}\right)_{m} \rightarrow \tilde{\Omega}^{q}\left(U_{\sigma}\right)_{c m}$ is multiplication by $c^{q} \chi^{(c-1) m}$. By (3.3), this is an isomorphism for all $c \neq 0$.

Proposition 6.4. For any toric $k$-variety $X$, the natural maps (3.4) induce isomorphisms, for all $q$ :

$$
\Omega^{q}(X)^{\mathfrak{c}} \rightarrow \tilde{\Omega}^{q}(X)^{\mathfrak{c}}
$$

Proof. We may assume $X=U_{\sigma}$, so that $\Omega^{q}(X)=\Omega_{k[A]}^{q}$ for $A=\sigma^{\vee} \cap M$. It suffices to check that the map is an isomorphism in each weight $m \in M_{\mathfrak{c}}$; without loss of generality, one may assume $m \in M$. By Lemma 3.5. $\left(\Omega_{k[A]}^{q}\right)_{m} \cong\left(\Omega_{k[B]}^{q}\right)_{m}$, where $B=A \cap \sigma(m)^{\perp}$. By Lemma 6.3, $\theta_{c}$ coincides with multiplication by $c^{q} \chi^{(c-1) m}$ both as a map $\left(\Omega_{k[A]}^{q}\right)_{m} \rightarrow\left(\Omega_{k[A]}^{q}\right)_{c m}$ and as a map $\left(\Omega_{k[B]}^{q}\right)_{m} \rightarrow\left(\Omega_{k[B]}^{q}\right)_{c m}$. Hence the group

$$
\Omega^{q}(X)_{m}^{\mathfrak{c}}=\lim _{\longrightarrow}\left(\left(\Omega_{k[A]}^{q}\right)_{m} \stackrel{\theta_{c_{1}}}{\longrightarrow}\left(\Omega_{k[A]}^{q}\right)_{c_{1} m} \stackrel{\theta_{c_{2}}}{\longrightarrow} \cdots\right)
$$


is the weight $m$ part of the localization of $\Omega_{k[B]}^{q}$ at $\chi^{m}$, i.e., of $\Omega^{q}\left(k[B]\left[\chi^{-m}\right]\right)$. By construction, $m$ is not on any proper face of $\sigma(m)^{\vee} \cap \sigma(m)^{\perp}$. By Lemma 6.2,

$$
\Omega^{q}\left(k[B]\left[\chi^{-m}\right]\right)_{m} \cong \Omega^{q}(k[B+\langle-m\rangle])_{m}=\Omega^{q}(k[T])_{m}, \quad T=M \cap \sigma(m)^{\perp} .
$$

Since $T$ is a free abelian group, $\left(\Omega_{k[T]}^{q}\right)_{m} \cong \bigwedge^{q}(T) \otimes k$. Now recall that by Remark 6.3 .1 and (3.3) we also have

$$
\left(\tilde{\Omega}_{k[T]}^{q}\right)_{m}^{\mathfrak{c}} \cong \tilde{\Omega}^{q}\left(U_{\sigma}\right)_{m}^{\mathfrak{c}}=\tilde{\Omega}^{q}\left(U_{\sigma}\right)_{m} \cong k \cdot \chi^{m} \otimes \bigwedge^{q}(T) .
$$

The map $\left(\Omega_{k[T]}^{q}\right)_{m} \rightarrow\left(\tilde{\Omega}_{k[T]}^{q}\right)_{m}$ is given by (3.4), and it is an isomorphism by inspection.

In order to prove an analogous result for Hochschild homology, we need to briefly review the decomposition of Hochschild homology into summands given by the (higher) André-Quillen homology groups. For more details, we refer the reader to [20, 3.5] or [27, 8.8].

For a commutative $k$-algebra $R$, one forms a simplicial polynomial $k$-algebra $R$. and a simplicial ring map $R_{\bullet} \rightarrow R$ which is a homotopy equivalence on underlying simplicial sets. The (higher) cotangent complex $\mathbb{L}_{X / k}^{(q)}$ is defined to be the simplicial $R$-module $R \otimes_{R_{\bullet}} \Omega_{R \bullet}^{q}$, and the André-Quillen homology groups of $R$ are defined to be $D_{p}^{(q)}(R)=H_{p}\left(\mathbb{L}_{X / k}^{(q)}\right)$. The $R$-modules $D_{p}^{(q)}(R)$ are independent up to isomorphism of the choices made.

In general, there is a natural spectral sequence of $R$-modules

$$
D_{p}^{(q)}(R) \Longrightarrow H H_{p+q}(R)
$$

and a natural $R$-module isomorphism $D_{0}^{(q)}(R) \cong \Omega_{R / k}^{q}$. Since we are assuming $\operatorname{char}(k)=0$, this spectral sequence degenerates to give a natural decomposition of $R$-modules

$$
H H_{n}(R) \cong \bigoplus_{p+q=n} D_{p}^{(q)}(R)=\Omega_{R / k}^{q} \oplus \bigoplus_{p+q=n, p>0} D_{p}^{(q)}(R)
$$

Since the André-Quillen homology groups are functorial for ring maps, the endomorphisms $\theta_{c_{i}}$ preserve this decomposition.

Lemma 6.5. Let $U_{\sigma}$ be an affine toric variety. Then the $D_{p}^{(q)}\left(U_{\sigma}\right)$ are $M$-graded modules and, for every $m \in \sigma^{\vee} \cap M$, the map $\theta_{c}: D_{p}^{(q)}\left(U_{\sigma}\right)_{m} \rightarrow D_{p}^{(q)}\left(U_{\sigma}\right)_{c m}$ is multiplication by $c^{q} \chi^{(c-1) m}$.

Proof. Let $A=\sigma^{\vee} \cap M$ and form a simplicial resolution of $A$ by free abelian monoids $A$. $\rightarrow A$. That is, $A$. is a simplicial abelian monoid which in each degree is free abelian and the map of simplicial abelian monoids $A_{\bullet} \rightarrow A$ is a homotopy equivalence. This is possible by the same basic cotriple resolution used to form simplicial free resolutions of $k$-algebras (see [27, 8.6]). For functorial reasons, $k\left[A_{\bullet}\right] \rightarrow k[A]$ is a free simplicial resolution of $k[A]$. We therefore have

$$
D_{p}^{(q)}(k[A])=H_{p}\left(k[A] \otimes_{k\left[A_{\bullet}\right]} \Omega_{k\left[A_{\bullet}\right]}^{q}\right) .
$$

For each $n$, the ring $k\left[A_{n}\right]$ is $M$-graded by the maps $\delta_{n}: A_{n} \rightarrow A \subset M$. Thus the simplicial ring $k\left[A_{\bullet}\right]$ is also $M$-graded, and the map $k\left[A_{\bullet}\right] \rightarrow k[A]$ of simplicial rings preserves this grading. It follows that $k[A] \otimes_{k\left[A_{\bullet}\right]} \Omega_{k\left[A_{\bullet}\right]}^{q}$ is naturally $M$-graded, 
where the weight of $\chi^{u_{0}} \otimes d\left(\chi^{u_{1}}\right) \wedge \cdots \wedge d\left(\chi^{u_{q}}\right)$ is $u_{0}+\delta_{n}\left(u_{1}\right)+\cdots+\delta_{n}\left(u_{q}\right)$, for any $u_{0} \in A$ and $u_{1}, \ldots, u_{q} \in A_{n}$. Hence $D_{p}^{(q)}(k[A])$ is an $M$-graded $k[A]$-module, and it is clear that, for any positive integer $c$, the endomorphism $\theta_{c}$ of $D_{p}^{(q)}(k[A])$ maps the weight $m$ summand to the weight $\mathrm{cm}$ summand. To prove that the map

$$
\theta_{c}: D_{p}^{(q)}(k[A])_{m} \rightarrow D_{p}^{(q)}(k[A])_{c m}
$$

coincides with multiplication by $c^{q} \chi^{(c-1) m}$, it suffices to prove the analogous assertion for the $M$-graded $k[A]$-modules $k[A] \otimes_{k\left[A_{n}\right]} \Omega_{k\left[A_{n}\right]}^{q}$. The proof of this is exactly like the proof of Lemma 6.3. using $\omega=\chi^{u_{0}} \otimes d \chi^{u_{1}} \wedge \cdots \wedge d \chi^{u_{q}}$.

Theorem 6.6. For any toric $k$-variety $X$, the natural maps

$$
\Omega^{q}(X)^{\mathfrak{c}} \rightarrow H H_{q}(X)^{\mathfrak{c}}
$$

are isomorphisms, for all $q$.

Proof. By the spectral sequence in Remark 6.1.1 we may assume that $X$ is affine, say of the form $X=U_{\sigma}$ for some cone $\sigma$. Setting $A=\sigma^{\vee} \cap M$, the coordinate ring of $X$ is $k[A]$. To establish the isomorphism $\Omega^{p}\left(U_{\sigma}\right)^{\mathfrak{c}} \cong H H_{p}\left(U_{\sigma}\right)^{\mathfrak{c}}$ it suffices to prove that

$$
D_{p}^{(q)}\left(k\left[\sigma^{\vee} \cap M\right]\right)^{\mathfrak{c}}=0
$$

for all $p>0$. As in the proof of Proposition 6.4, it suffices to fix an arbitrary $m \in M$ and show that the weight $m$ part vanishes. By Lemma 3.5, $D_{p}^{(q)}(k[A])_{m} \cong$ $D_{p}^{(q)}(k[B])_{m}$, where $B=A \cap \sigma(m)^{\perp}$. By Lemma 6.5 $\theta_{c}$ coincides with multiplication by $c^{q} \chi^{(c-1) m}$ both as a map $D_{p}^{(q)}(k[A])_{m} \rightarrow D_{p}^{(q)}(k[A])_{c m}$ and as a map $D_{p}^{(q)}(k[B])_{m} \rightarrow D_{p}^{(q)}(k[B])_{c m}$. Hence the weight $m$ summand

$$
D_{p}^{(q)}(X)_{m}^{\mathfrak{c}}=\lim _{\longrightarrow}\left(D_{p}^{(q)}(k[A])_{m} \stackrel{\theta_{c_{1}}}{\longrightarrow} D_{p}^{(q)}(k[A])_{c_{1} m} \stackrel{\theta_{c_{2}}}{\longrightarrow} \cdots\right)
$$

is the weight $m$ part of the localization of $D_{p}^{(q)}(k[B])$ at $\chi^{m}$, i.e., of $D_{p}^{(q)}\left(k[B]\left[\chi^{-m}\right]\right)$.

Recall that $\sigma(m) \subset \sigma$ denotes the face of $\sigma$ (possibly just the origin) on which $m=0$. By Lemma 6.2.

$$
D_{p}^{(q)}\left(k[B]\left[\chi^{-m}\right]\right)_{m} \cong D_{p}^{(q)}(k[B+\langle-m\rangle])_{m}=D_{p}^{(q)}(k[T])_{m}, \quad T=M \cap \sigma(m)^{\perp} .
$$

Since $T=M \cap \sigma(m)^{\perp}$ is a free abelian group, we have

$$
D_{p}^{(q)}\left(k[B]\left[\frac{1}{\chi^{m}}\right]\right)=D_{p}^{(q)}(k[T])=0
$$

for all $p>0$. This proves that $D_{p}^{(q)}(k[A])^{\mathfrak{c}}=0$ for all $p>0$, proving the theorem.

Corollary 6.7. For any field $k$ of characteristic 0 and any toric $k$-variety $X$, we have a natural isomorphism for all $n$ :

$$
H H_{n}(X / \mathbb{Q})^{\mathfrak{c}} \stackrel{\simeq}{\longrightarrow} \mathbb{H}_{c d h}^{-n}(X, H H(-/ \mathbb{Q}))^{\mathfrak{c}} .
$$

The right hand side of Corollary 6.7 denotes Hochschild homology with $c d h$ descent imposed (and localized by $\mathfrak{c}$ ). (On both sides, we take Hochschild homology over $\mathbb{Q}$.) 
Proof. Let us write $X_{\mathbb{Q}}$ for the model of $X$ defined over the rationals and $X_{k}=X$ for the model over $k$. We have $X_{k}=X_{\mathbb{Q}} \times_{\operatorname{Spec} \mathbb{Q}} \operatorname{Spec} k$.

The natural map

$$
H H_{n}\left(X_{k / k}\right)^{\mathfrak{c}} \longrightarrow \mathbb{H}_{\mathrm{cdh}}^{-n}(X, H H)^{\mathfrak{c}}
$$

is an isomorphism. Since both sides satisfy Zariski descent, this is an immediate consequence of Theorem 4.1 and Theorem 6.6. The Künneth formula for Hochschild homology, described before Corollary 4.5, gives

$$
H H_{*}(X / \mathbb{Q})^{\mathfrak{c}} \cong H H_{*}\left(X_{\mathbb{Q}} / \mathbb{Q}\right)^{\mathfrak{c}} \otimes_{\mathbb{Q}} \Omega_{k / \mathbb{Q}}^{*}
$$

In particular, one gets long exact sequences for $H H_{*}(-/ \mathbb{Q})^{\mathfrak{c}}$ associated to abstract blow-ups of toric $k$-varieties. Since the map

$$
H H_{n}\left(X_{k} / \mathbb{Q}\right)^{\mathfrak{c}} \cong \mathbb{H}_{\text {cdh }}^{-n}(X, H H(-/ \mathbb{Q}))^{\mathfrak{c}}
$$

is an isomorphism whenever $X$ is smooth by $[3,2.4]$, the result holds by induction and the five-lemma.

Corollary 6.8. For any field $k$ of characteristic 0 and any toric $k$-variety $X$, and all $n$, we have

$$
H C_{n}(X / \mathbb{Q})^{\mathfrak{c}} \cong \mathbb{H}_{c d h}^{-n}(X, H C(-/ \mathbb{Q}))^{\mathfrak{c}} .
$$

Proof. There is a map from the SBI sequence for $H H$ and $H C$ to the SBI sequence for its $c d h$-fibrant variant. Applying the exact functor $(-)^{\mathfrak{c}}$ yields a similar map of long exact sequences, every third term of which is the isomorphism of Corollary 6.7 The result now follows by induction on $n$, since all complexes are cohomologically bounded above.

Theorem 6.9. For any field $k$ of characteristic 0 and any toric $k$-variety $X$, we have

$$
K_{*}(X)^{\mathfrak{c}} \cong K H_{*}(X)^{\mathfrak{c}} .
$$

Proof. Since $(-)^{\mathfrak{c}}$ is exact, it suffices by Theorem 5.5 to show that $H_{\text {Zar }}^{*}\left(X, \mathcal{F}_{H C}\right)^{\mathfrak{c}}$ vanishes. Again because $(-)^{\mathfrak{c}}$ is exact, we have a long exact sequence

$$
\cdots \rightarrow H_{\text {Zar }}^{n}\left(X, \mathcal{F}_{H C}\right)^{\mathfrak{c}} \rightarrow H C_{-n}(X / \mathbb{Q})^{\mathfrak{c}} \rightarrow \mathbb{H}_{\text {cdh }}^{n}(X, H C(-/ \mathbb{Q}))^{\mathfrak{c}} .
$$

The desired vanishing follows from the previous corollary.

Corollary 6.10 (Gubeladze's Dilation Theorem). Let $\Gamma$ be an arbitrary commutative, cancellative, torsionfree monoid without non-trivial units. Then for every sequence $\mathfrak{c}$ and every $p,\left(K_{p}(k[\Gamma]) / K_{p}(k)\right)^{\mathfrak{c}}=0$.

Proof. To prove the Dilation Theorem, it suffices to prove it for all "affine positive normal" monoids, i.e., for monoids of the form $\Gamma=\sigma^{\vee} \cap M$ such that $\sigma^{\perp}=0$. This is a reformulation of [12, 3.4], and is stated explicitly in [15, Proposition 2.1] (up to the typo where $K_{p}(R[M])$ should be $\left.K_{p}(R[M]) / K_{p}(R)\right)$.

For such $\Gamma, X=\operatorname{Spec}(k[\Gamma])$ is a toric variety, and the proof of Proposition 5.6 above shows that $k[\Gamma]$ is $\mathbb{N}$-graded with $k$ in weight 0 . Hence $K H(X) \simeq K(\operatorname{Spec} k)$. The result now follows from Theorem 6.9.

Remark 6.11. In [16, Gubeladze proves an unstable version of his Dilation Theorem for the groups $K_{1}$ and $K_{2}$, which is valid for any regular coefficient ring in place of the field $k$. In [17, he proves that his Dilation Theorem remains valid if one replaces the field $k$ by any regular coefficient ring that contains a copy of $\mathbb{Q}$. 


\section{ACKNOWLEDGEMENTS}

The third author thanks Joseph Gubeladze and Srikanth Iyengar for useful conversations that contributed to this paper.

\section{REFERENCES}

[1] G. Cortiñas, The obstruction to excision in K-theory and cyclic homology, Invent. Math. 454 (2006), 143-173. MR2207785 (2006k:19006)

[2] G. Cortiñas, C. Haesemeyer, M. Schlichting and C. Weibel, Cyclic homology, $c d h$-cohomology and negative $K$-theory, Annals of Math. 167 (2008), 549-573. MR2415380

[3] G. Cortiñas, C. Haesemeyer and C. Weibel, $K$-regularity, cdh-fibrant Hochschild homology, and a conjecture of Vorst, J. Amer. Math. Soc., 21 (2008), no. 2, 547-561. MR2373359

[4] G. Cortiñas, C. Haesemeyer and C. Weibel, Infinitesimal cohomology and the Chern character to negative cyclic homology, Preprint, available at http://www.math.uiuc.edu/Ktheory/0824/, 2007.

[5] V. Danilov, The geometry of toric varieties, Russian Math. Surveys 33 (1978), 97-154. MR 495499 (80g:14001)

[6] V. Danilov, de Rham complex on toroidal variety, pp. 26-38 in Lecture Notes in Math. 1479, Springer, 1991. MR1181204 (93i:14050)

[7] P. Deligne, Théorie de Hodge: III, Publ. Math. IHES 44 (1974), 5-77. MR0498552 (58:16653b)

[8] Ph. DuBois, Complexe de de Rham filtré d'une variété singulière, Bull. Soc. Math. France 109 (1981), 41-81. MR613848 (82j:14006)

[9] W. Fulton, Introduction to Toric Varieties, Annals Math. Study 131, Princeton Univ. Press, 1993. MR1234037 (94g:14028)

[10] F. Guillén and V. Navarro-Aznar, Un critère d'extension des foncteurs définis sur les schémas lisses, Publ. Math. IHES 95 (2002), 1-91. MR1953190(2004i:14020)

[11] J. Gubeladze, Anderson's conjecture and the maximal class of monoids over which projective modules are free, Math. USSR Sb. 135 (1988), 169-181 (Russian). MR937805 (89d:13010)

[12] J. Gubeladze, Nontriviality of $S K_{1}(R[M])$, J. Pure Applied Alg. 104 (1995), 169-190. MR.1360174 (96j:19002)

[13] J. Gubeladze, Higher $K$-theory of toric varieties $K$-Theory 28 (2003), 285-327. MR 2017618 (2004i:19004)

[14] J. Gubeladze, Toric varieties with huge Grothendieck group, Adv. Math. 186 (2004), 117-124. MR2065508 (2005d:14014)

[15] J. Gubeladze, The nilpotence conjecture in $K$-theory of toric varieties, Inventiones Math. 160 (2005), 173-216. MR2129712 (2006d:14057)

[16] J. Gubeladze, The Steinberg group of a monoid ring, nilpotence, and algorithms, J. Algebra, 307 (2007), 461-496. MR 2278067 (2007i:19003)

[17] J. Gubeladze, Global coefficient ring in the nilpotence conjecture, Proc. Amer. Math. Soc. 136 (2008), no. 2, 499-503. MR2358489

[18] C. Haesemeyer, Descent properties of homotopy K-theory, Duke Math. J. 125 (2004), 589620. MR2166754 (2006g:19002)

[19] M.-N. Ishida, Torus embeddings and de Rham complexes, pp. 111-145 in Adv. Stud. Pure Math., 11, North-Holland, Amsterdam, 1987. MR951199 (89j:14028)

[20] J.-L. Loday, Cyclic homology. Springer-Verlag, Berlin, 1992. MR.1217970 (94a:19004)

[21] A. Suslin and V. Voevodsky, Bloch-Kato conjecture and motivic cohomology with finite coefficients, pp. 117-189 in The arithmetic and geometry of algebraic cycles (Banff, AB, 1998), volume 548 of NATO Sci. Ser. C Math. Phys. Sci.. Kluwer, Dordrecht, 2000. MR 1744945 (2001g:14031)

[22] R. Swan, Gubeladze's proof of Anderson's conjecture, Contemp. Math. 124, Amer. Math. Soc., Providence, RI, 1992. MR1144038 (92m:13012)

[23] W. Vasconcelos, A note on normality and module of differentials, Math. Z. 105 (1968), 291293. MR 0227165 (37:2750)

[24] C. Weibel and S. Geller, Étale descent for Hochschild and cyclic homology, Comment. Math. Helvetici 66 (1991), 368-388. MR1120653 (92e:19006) 
[25] C. Weibel, Module structures on the K-theory of graded rings, J. Algebra 105 (1987), 465-483. MR873680 (88f:18018)

[26] C. Weibel, Homotopy algebraic K-theory, AMS Contemp Math. 83 (1989), 461-488. MR991991 (90d:18006)

[27] C. Weibel, An introduction to homological algebra, Cambridge Univ. Press, 1994. MR 1269324 (95f:18001)

[28] C. Weibel, Cyclic homology of schemes, Proc. AMS 124 (1996), 1655-1662. MR.1277141 (96h:19003)

Departamento de Matemática, FCEyn-UBA, Ciudad Universitaria Pab 1, 1428 Buenos Aires, Argentina - and - Departamento Álgebra, Fac. de Ciencias, Prado de la MagDALENA S/N, 47005 VAlLAdOLID, Spain

E-mail address: gcorti@dm.uba.ar

Department of Mathematics, University of Illinois, Urbana, Illinois 61801

E-mail address: chh@math.uiuc.edu

Current address: Department of Mathematics, University of California, Los Angeles, Box 95155, Los Angeles, California 90095-1555

E-mail address: chh@math.ucla.edu

Department of Mathematics, University of Nebraska, Lincoln, Lincoln, Nebraska 68588-0130

E-mail address: mwalker5@math.unl.edu

Department of Mathematics, Rutgers University, New Brunswick, New Jersey 08901

E-mail address: weibel@math.rutgers.edu 